\title{
Frecuencia de anticuerpos contra el virus de la leucosis bovina (VLB) en leche de tanque
}

\author{
Ricardo García $\mathrm{N}^{1}{ }^{\otimes}$ M.Sc; Martha Olivera-Angel ${ }^{1,2, *}{ }^{凶}$ Dr.Sci.Agr.
}

\begin{abstract}
${ }^{1}$ Universidad de Antioquia, Facultad de Ciencias Agrarias, Unidad de Diagnóstico, Medellín, Colombia.
2 Universidad de Antioquia, Facultad de Ciencias Agrarias, Grupo de investigación Biogénesis, Medellín, Colombia

*Correspondence: martha.olivera@udea.edu.co
\end{abstract}

Recibido: Febrero 2018; Aceptado: Marzo 2019; Publicado: Septiembre 2019.

\section{RESUMEN}

Objetivo. Determinar presencia de anticuerpos contra VLB en muestras de leche de tanques, en varios departamentos de Colombia. Materiales y métodos. De 2220 muestras llegadas al laboratorio de calidad de la leche de la Universidad de Antioquia para análisis de calidad para pago de una quincena en el mes de mayo de 2016, se seleccionaron 329 al azar y de manera ponderada según el número de muestras disponibles para cada uno de los 7 departamentos participantes. Estas muestras fueron sometidas a la prueba de ELISA para determinar la presencia de anticuerpos contra VLB. Resultados. El resultado principal de este análisis al azar de tanques de leche en busca de anticuerpos contra BLV, fue la positividad de los predios en rangos que oscilan entre el 57 y el 100\%; el abordaje de este problema de salud bovina en Colombia es posible mediante las muestras de leche de tanque que rutinariamente llegan a los laboratorios de análisis de calidad. Conclusiones. La infección con BLV está presente en Colombia, el establecimiento de programas de control se podría hacer aprovechando las muestras de leche que se envían de rutina a los laboratorios acreditados para pago.

Palabras clave. ELISA, predio, Colombia, laboratorio calidad de leche, tanque leche (Fuente: AGROVOC).

\section{ABSTRACT}

Objective. To determine the presence of anti-BLV antibodies in samples from bulk tank milk from several Colombian states. Materials and methods. Out of 2220 samples arriving to the quality milk laboratory from the University of Antioquia in a fortnight period from the month of may 2016, 329 were randomly selected according to the number of samples per each one of the 7 states participating in the study. These samples were run through ELISA test for anti-BLV antibodies. Results. The main result of this random analysis of milk tanks in search of antibodies against BLV was the positivity of the farms in ranks ranging from 57 to $100 \%$; the approach to this bovine health problem in Colombia is possible through the tank milk samples that routinely reach the quality analysis laboratories. Conclusions. The infection with BLV is present in Colombia, the establishment of control programs could be done taking advantage of the samples of milk that are sent routinely to the laboratories accredited for payment.

Keywords: ELISA, milk bulk tank, farm, Colombia, Quality Milk laboratory (Source: AGROVOC).

Como citar (Vancouver)

García NR, Olivera-Angel M. Frecuencia de anticuerpos contra el virus de la leucosis bovina (VLB) en leche de tanque. Rev MVZ Cordoba. 2019; 24(3):73627365. DOI: https://doi.org/10.21897/rmvz.1839 (c) (1) () (1) (los) autor (es), Revista MVZ Córdoba 2019. Este artículo se distribuye bajo los términos de la licencia internacional Creative Commons obra de modo no comercial, siempre y cuando den crédito y licencien sus nuevas creaciones bajo las mismas condiciones. 


\section{INTRODUCCIÓN}

La leucosis enzoótica bovina causada por el virus de la leucemia bovina, es una enfermedad de importancia económica: los animales infectados mueren o deben ser descartados precozmente por su baja producción lechera (1) sin que su carne pueda ser aprovechada por la presencia de linfosarcomas (2). Adicionalmente se ha demostrado la presencia del virus en tejido mamario en humanos si bien el potencial zoonótico este virus no ha sido determinado con claridad $(3,4)$.

La prevalencia de esta infección en América del Norte ha ido en aumento hasta llegar a un $90 \%$ en Canadá (2) y al $94.2 \%$ en Estados Unidos (5). En Argentina, en 2001, la prevalencia de hato en vacas lecheras fue del $84 \%$ (6), mientras que en Perú, en 2015, encontraron una seroprevalencia del $92.7 \%$ en un hato lechero ( 7 ). En Colombia, en suero sanguíneo, en 2016, en diferentes estudios, se informó una prevalencia que fluctuó en un rango de 3.9 y $73 \%$ en diferentes regiones (8-12).

Los países europeos han logrado la erradicación de la enfermedad, usando como herramienta el análisis de leche de tanque, gracias a que se trata de un método no invasivo, fácil y de bajo costo Con esta estrategia se puede realizar la vigilancia epidemiológica y también monitorear la erradicación (13). El objetivo de este estudio fue determinar en leche de tanque, la prevalencia de predios positivos a anticuerpos para LVB, en algunos departamentos colombianos.

\section{MATERIALES Y MÉTODOS}

Tipo de estudio. Se realizó un estudio de corte transversal para evaluar la presencia de anticuerpos contra el virus de la leucosis bovina en algunos predios de ganaderías de leche. En un total de 2200 muestras de leche que llegaron en una quincena para análisis de calidad y con un margen de error del $5 \%$ y un nivel de confianza del $95 \%$ se analizaron 329 de 7 departamentos.

La distribución de muestras por departamento se realizó con base en el porcentaje de muestras que llegaron en el periodo dicho, así: el 55\% de las muestras correspondió al departamento de Cundinamarca, el $12 \%$ para el Meta, el $11 \%$ para César, el 7\% para Antioquia; Boyacá, Nariño y Valle representaron el 3 y $4 \%$ del total de muestras analizadas (Tabla 1).

Aspectos éticos. El nombre de la empresa, el nombre de los predios, así como los resultados del ELISA no se muestran para proteger la confidencialidad de la empresa y de los ganaderos. Este trabajo se realizó con el consentimiento informado y acuerdo de confidencialidad de los participantes.
Tabla 1. Distribución del porcentaje de predios muestreados por departamento.

\begin{tabular}{|c|c|c|}
\hline Departamento & Total Predios & $\%$ Predios \\
\hline Antioquia & 26 & 7.9 \\
\hline Boyacá & 13 & 4.0 \\
\hline Cesar & 39 & 11.9 \\
\hline Cundinamarca & 184 & 55.9 \\
\hline Meta & 42 & 12.8 \\
\hline Nariño & 14 & 4.3 \\
\hline Valle & 11 & 3.3 \\
\hline Total & 329 & 100 \\
\hline
\end{tabular}

Método de laboratorio. Se utilizó una prueba de ELISA indirecta Leukosis Milk Screening $A b$ (IDEXX`), que detecta anticuerpos anti VLB presentes en leche. La lectura se realizó por espectrofotometría $(450 \mathrm{~nm})$ usando el software $\mathrm{X}$-Check Plus y se expresó en porcentaje de positividad (M/P\%) (DO muestra/DO de la media del control positivo) x 100, donde DO es densidad óptica. Muestras con M/P inferior o igual a $60 \%$ se considera negativo, muestras con un M/P superior a $60 \%$ e inferior a $70 \%$ se consideran dudosas y muestras con un $\mathrm{M} / \mathrm{P}$ superior a $70 \%$ se consideran positivas. La especificidad de la técnica reportada por la casa productora es del $98.8 \%$, pero no indica dato de sensibilidad debido a la dificultad para obtener sueros verdaderamente positivos.

Análisis de resultados. El análisis estadístico descriptivo se llevó a cabo con el programa estadístico SPSS versión 24 con licencia para la Universidad de Antioquia y los resultados se expresan en la tabla de frecuencias 2.

\section{RESULTADOS}

Resultados totales y por departamento. En la tabla 2, se presentan el número y el porcentaje de predios positivos a anticuerpos contra VLB por departamento. Es notorio que todos los departamentos incluidos en el estudio fueron positivos.

Tabla 2. Resultados de positividad y negatividad a anticuerpos contra VLB por departamento

\begin{tabular}{cccccc}
\hline Departamento & $\begin{array}{c}\text { Total } \\
\text { Predios }\end{array}$ & $\begin{array}{c}\text { Predios } \\
\text { Positivos }\end{array}$ & $\begin{array}{c}\text { \% } \\
\text { Positivos }\end{array}$ & $\begin{array}{c}\text { Predios } \\
\text { Negativos }\end{array}$ & $\begin{array}{c}\% \\
\text { Negativos }\end{array}$ \\
\hline Antioquia & 26 & 25 & 96 & 1 & 4 \\
Boyacá & 13 & 9 & 69 & 4 & 31 \\
Cesar & 39 & 39 & 100 & 0 & 0 \\
Cundinamarca & 184 & 163 & 89 & 21 & 11 \\
Meta & 42 & 24 & 57 & 18 & 43 \\
Nariño & 14 & 11 & 79 & 3 & 21 \\
Valle & 11 & 10 & 91 & 1 & 9 \\
\hline Total & $\mathbf{3 2 9}$ & $\mathbf{2 8 1}$ & $\mathbf{8 5}$ & $\mathbf{4 8}$ & $\mathbf{1 5}$ \\
\hline
\end{tabular}




\section{DISCUSIÓN}

El resultado principal de este análisis al azar de tanques de leche en busca de anticuerpos contra BLV, fue la positividad de los predios en rangos que oscilan entre el 57 y el $100 \%$. Esto concuerda con los hallazgos de seroprevalencia en Colombia (8-12), si bien los porcentajes de positividad son mayores en nuestro estudio. En Estados Unidos y Canadá, estudios realizados en muestras de tanque, reportan niveles de positividad mayores a los reportados como promedio en este informe $(2,5)$.

Por tratarse de un estudio de tipo exploratorio, las inferencias que se puede hacer son pocas. Sin embargo, consideramos que queda claro que el abordaje de este problema de salud bovina en Colombia es posible mediante las muestras de leche de tanque que rutinariamente llegan a los laboratorios de análisis de calidad. Esta estrategia fue analizada por Reber et al (13) y en su estudio concluyen que el uso de muestras de leche de tanque mejora la flexibilidad para la supervisión de los programas de vigilancia y control; que además se puede mejorar la intensidad de la vigilancia y se pueden detectar rápidamente predios recientemente infectados (13). Por otra parte Dereń et al (14) sugieren que, la posibilidad de hacer el seguimiento geográfico mediante el estudio de los tanques puede contribuir a la efectiva erradicación de la enfermedad. La iniciación de un programa de control comienza con el establecimiento de la prevalencia de anticuerpos y según algunos autores Dereń et al (14) y Lee et al (15), el seguimiento bimestral, con análisis de tanques y con la toma de las medidas sanitarias apropiadas se puede llegar a la erradicación en 10 años (14).

Es importante recordar que al tanque llega la leche de las vacas que se están ordeñando ese día; o sea que no está presente la muestra de las vacas secas, enfermas, o recién paridas, por tanto las tomas se deben hacer con dos meses de intervalo (13). Estos últimos autores desarrollaron un modelo o algoritmo que permite estimar con cierta precisión el número de vacas infectadas en cada predio $(14,15)$.

En Colombia, previo consentimiento informado, como lo hemos hecho en este estudio piloto, que es el primero en su género en el país, se podría adoptar esta estrategia para el establecimiento de un programa nacional de control de esta infección.

\section{Conflicto de intereses}

Los autores del presente estudio declaramos que no existe conflicto de intereses con la publicación de este manuscrito.

\section{Agradecimientos}

Los autores de esta investigación agradecen a la Universidad de Antioquia, a la empresa AQUALAB SAS por facilitarnos el kit de diagnóstico en matriz leche y a los centros de acopio de los diferentes departamentos del país.

\section{REFERENCIAS}

1. Bartlett PC, Sordillo LM, Byrem TM, Norby B, Grooms DL, Swenson CL, et al. Options for the control of bovine leukemia virus in dairy cattle. J Am Vet Med Assoc. 2014; 244(8):914-922. https://doi.org/10.2460/ javma.244.8.914

2. Nekouei O, Durocher J, Keefe G. Diagnostic performance of an indirect enzyme-linked immunosorbent assay (ELISA) to detect bovine leukemia virus antibodies in bulk-tank milk samples. Can Vet J. 2016; 57(7):778780. https://www.ncbi.nlm.nih.gov/pmc/ articles/PMC4904818/

3. Buehring GC, Shen HM, Jensen HM, Jin $D L$, Hudes M, Block G. Exposure to bovine leukemia virus is associated with breast cancer: a case-control study. PloS one. 2015; 10(9):e0134304. https://doi.org/10.1371/ journal.pone. 0134304
4. Buehring GC, Shen $H$, Schwartz DA, Lawson JS. Bovine leukemia virus linked to breast cancer in Australian women and identified before breast cancer development. PloS one. $2017 ; 12(6)$ :e0179367. https://doi. org/10.1371/journal.pone.0179367

5. LaDronka RM, Ainsworth $S$, Wilkins $M J$, Norby B, Byrem TM, Bartlett PC. Prevalence of Bovine Leukemia Virus Antibodies in US Dairy Cattle. Vet Med Int. 2018; 2018(Article ID 5831278):1-8. https://doi. org/10.1155/2018/5831278

6. Trono KG, Pérez-Filgueira DM, Duffy $S$, Borca MV, Carrillo C. Seroprevalence of bovine leukemia virus in dairy cattle in Argentina: comparison of sensitivity and specificity of different detection methods. Vet Microbiol. 2001; 83(3):235-248. https://doi. org/10.1016/s0378-1135(01)00420-5 
7. Sandoval R, Delgado A, Ruiz L, Ramos O. Determinación de la seroprevalencia del virus de la Leucemia Bovina en un establo lechero de Lima, Perú. Rev Investig Vet Peru. 2015; 26(1):152-158. https://doi.org/10.15381/ rivep.v26i1.10919

8. Meza-Barreto G, Sanjuanelo-Corredor DW, Gallego-Marín MI. Detección molecular del virus de la leucosis bovina: un estudio por conglomerados en Colombia. Ciencia y Agricultura. 2016; 13(2):47-55. https://doi. org/10.19053/01228420.v13.n2.2016.5552

9. Hernández D, Muñoz J, Álvarez L. Asociación del locus BOLA-DRB3. 2 con el virus de la leucosis bovina en el ganado criollo colombiano. Rev Colombiana Cienc Anim. 2014; 6(2):319326. https://doi.org/10.24188/recia. v6.n2.2014.435

10. Cadavid L. Impacto del virus de la leucosis bovina en la producción de leche. [Thesis de Maestría]. Colombia: Universidad Nacional de Colombia; 2012. http://bdigital.unal.edu. co/9308/

11. Rojas JLC, Martinez FA, Tarazona A, Cepeda BM. Prevalencia de la seropositividad a la leucosis bovina mediante la técnica diagnóstica de ELISA indirecta en hatos lecheros situados en Mesa de los Santos, Santander. Spei Domus. 2009; 5(11):6-11. https://revistas.ucc.edu.co/index.php/sp/ article/view/590
12. Bautista NA, Nova YA, Pulido-Medellín MO, Andrade-Becerra RJ. Determinación serológica de leucosis bovina enzoótica en novillas de levante y vacas adultas de la vereda Morichal, Yopal, Casanare. Ciencia y Agricultura. 2013; 10(1):31-37. https://doi. org/10.19053/01228420.2832

13. Reber A, Reist M, Schwermer H. Costeffectiveness of bulk-tank milk testing for surveys to demonstrate freedom from infectious bovine rhinotracheitis and bovine enzootic leucosis in Switzerland. Schweiz Arch Tierheilkd. 2012; 154(5):189-197. https:// doi.org/10.1024/0036-7281/a000329

14. Dereń W, Szewczyk A, Rułka J. The eradication of enzootic bovine leucosis in a large farm population. Pol J Vet Sci. 2003; 6(3):12-14. https://www.ncbi.nlm.nih.gov/ pubmed/14509351

15. Lee E, Kim EJ, Ratthanophart J, Vitoonpong R, $\mathrm{Kim} \mathrm{BH}$, Cho IS, et al. Molecular epidemiological and serological studies of bovine leukemia virus (BLV) infection in Thailand cattle. Infect Genet Evol. 2016; 41(July 2016):245-254. https:// doi.org/10.1016/j.meegid.2016.04.010 